\title{
Enhanced dissolution and solubility of Epalrestat with $\beta$-Cyclodextrin ternary complex using Arginine
}

\author{
Jagtap Sneha*1, Magdum Chandrakant ${ }^{2}$ \\ 1 Annasaheb Dange College of B Pharmacy, Ashta, Sangli - 416301 Maharashtra, India. \\ 2 Rajarambapu College of Pharmacy, Kasegaon, Sangli -415404 Maharashtra, India.
}

\begin{abstract}
The objective of present work is to achieve improvement in solubility and dissolution using Epalrestat with $\beta$-cyclodextrin ternary complex with addition of L-arginine. Kneading method with and without incorporation of L-arginine was used to obtain the solid systems (binary and ternary complex) of Epalrestat with $\beta$-cyclodextrin and characterized for DSC, PXRD, SEM. Phase solubility, saturation solubility, dissolution and stability studies were carried out. The effect of L-arginine was investigated on complexation efficiency of $\beta$-cyclodextrin towards Epalrestat in an aqueous media through phase solubility according to Higuchi and Connors. Phase solubility studies showed $A_{L}$ (linear) type of solubility curve in presence of L-arginine, the complexation efficiency and association constant of $\beta$-cyclodextrin towards Epalrestat were promoted by L-arginine signifying its use as a ternary component. Dissolution rate of Epalrestat and solubility were significantly improved by complexation with $\beta$-cyclodextrin as compared to Epalrestat alone. Ternary complex incorporated with L-arginine proved better than binary complex. Hence, L-arginine could be exploited as a ternary component to improve the solubility of Epalrestat via $\beta$-cyclodextrin complexation.
\end{abstract}

Keywords: Epalrestat, solubility enhancement, inclusion complexes, $\beta$-cyclodextrin, L-arginine.

Article Info: Received 26 Sep 2018; Review Completed 24 Oct 2018; Accepted 25 Oct 2018; Available online 15 Nov 2018

Cite this article as:

Jagtap S, Magdum C, Enhanced dissolution and solubility of Epalrestat with $\beta$-Cyclodextrin ternary complex using Arginine, Journal of Drug Delivery and Therapeutics. 2018; 8(6):62-67

DOI: http://dx.doi.org/10.22270/jddt.v8i6.2014

*Address for Correspondence:

Jagtap Sneha, Annasaheb Dange College of B Pharmacy, Ashta, Sangli - 416301 Maharashtra, India.

\section{INTRODUCTION}

Epalrestat (EPL), 2-[(5Z)-5-[(E)-2-methyl-3-phenylprop-2enylidene]-4-oxo-2-sulfanylidene-1,3-thiazolidin-3yl]acetic acid is a water-insoluble drug $(14 \mu \mathrm{M})$ that inhibits aldose reductase. ${ }^{1}$ The most common long-term complication in patients with diabetes mellitus is diabetic neuropathy. ${ }^{2}$ And EPL is used in its treatment. ${ }^{3}$ in hyperglycemic conditions; EPL reduces intracellular sorbitol accumulation, which can be efficacious in treating diabetic neuropathy. Main complications in patients with diabetes mellitus are neuropathy, retinopathy and nephropathy. 4,5 Cyclodextrins (CDs) have mainly been used as complexing agents to increase aqueous solubility, bioavailability and stability of drugs by the pharmaceutical industries. CDs are cyclic $\alpha-(1,4)$-linked oligosaccharides of $\alpha$-D-glucopyranose containing a relatively hydrophobic central cavity and a hydrophilic external surface.6,7 In present research paper, we proposed to improve solubility and the dissolution behavior of EPL by $\beta$ - cyclodextrin (CD) and its ternary complex with L-arginine (ARG). CDs have the ability to form inclusion complex with many drug molecules entrapping the guest molecule partially or completely within the cyclodextrin cavity thus resulting in an increase in the solubility of the drug. First, phase solubility studies were performed, which confirmed complexation of $\beta-C D$ and EPL in aqueous solution. Numerous investigations reported that, incorporation of small quantity of hydrophilic excipients such as organic acids, amino acids, basic compounds, and polymers to an aqueous complexation medium usually give rise to an enhancement in the complexation efficiency of cyclodextrins and ultimately reduce their amounts in pharmaceutical formulations. ${ }^{8}$ The purpose of the current study was to ameliorate the aqueous solubility, dissolution rate and thus bioavailability of EPL and this is achieved by ternary complexation of $\beta$-CD with EPL employing amino acids. ${ }^{9-13}$ The solubility of EPL in absence of $\beta$-CD, Intrinsic solubility $\left(\mathrm{S}_{0}\right)$ was determined. Complexation with ARG also promoted apparent stability constant (Ks) of ternary as compared to binary complex. The basic amino acid, ARG was proved to be a better option as an auxiliary substance to increase complexation efficiency (CE) of CDs in ternary complex. 
<smiles>CC(/C=C1\SC(=S)N(CC(=O)O)C1=O)=C\c1ccccc1</smiles>

Figure 1: Chemical Structure of EPL

Thus considering the upper hand advantage of adding amino acid as a ternary component during complexation of drug with $\beta$-CD, the present investigation was carried out to contribute the promotion of the solubility of poorly water-soluble drug, EPL via $\beta$-CD complexation using ARG as an auxiliary substance, which to our knowledge has not been reported yet.

\section{MATERIALS AND METHODS}

\subsection{Materials}

Epalrestat was a generous gift from Zydus Cadila Ahmadabad (India). $\beta$-CD and ARG were purchased from unique chemicals. All other reagents and solvents used were of analytical grade.

\subsection{Analysis of Epalrestat using a UV-visible spectrophotometer}

First, $100 \mu \mathrm{g} / \mathrm{ml}$ stock solution of Epalrestat was prepared in methanol and further dilutions were made in distilled water ranging from $03-18 \mu \mathrm{g} / \mathrm{ml}$. The calibration curve was obtained by recording the absorbance on a UV spectrophotometer (Shimadzu UV spectrophotometer 1800 ) at $390 \mathrm{~nm} .{ }^{14}$ Linearity in the calibration curve was observed with regression $\mathrm{R}^{2}=0.994$.

\subsection{Phase solubility studies}

The solubility behavior of EPL was examined in distilled water at room temperature $\left(25 \pm 2{ }^{\circ} \mathrm{C}\right)$ according to the method described by (Higuchi and Connors 1965) [15]. Excess amount of EPL(50mg) was added to $20 \mathrm{~mL}$ of aqueous solutions containing various concentrations of $\beta$ $\mathrm{CD}(1 \mathrm{mM}-10 \mathrm{mM})$ with or without addition of ARG $(0.25 \%$ $\mathrm{w} / \mathrm{v})$. The suspensions were mechanically shaken subsequently on rotary shaker for $72 \mathrm{~h}$ at $125 \mathrm{rpm}$ until equilibrium was achieved. The samples were filtered through Whatman filter paper 41, diluted if necessary and analyzed spectrophotometrically (Shimadzu UV spectrophotometer 1800) at $390 \mathrm{~nm}$. The association constant (Ks) of complex and CE of $\beta$-CD were calculated according to the equations (1) and (2) respectively 1,16 .

\section{Ks = Slope $/ S_{0}$ (1-Slope)}

$S_{0}$ is the solubility of EPL in absence of $\beta$-CD and slope is obtained from the phase solubility diagram obtained by plotting moles of drug on $y$-axis and moles of $\beta$-CD on $x-$ axis. It gives idea about the linear dependence of drug concentration to $\beta$-CD concentration, with slope ratio below one usually assumes $1: 1$ ratio of the complex and refers to $A_{L}$ (linear) type of the phase solubility curve.

CE $=$ SO.K 1:1 = Slope / (1-Slope)

\subsection{Preparation of Binary and kneaded ternary complexes}

Binary complex (BC) - For binary complex EPL and $\beta$-CD were weighed accurately, mixed thoroughly by triturating in a mortar and sieved through a 120 sieve. Binary complex were stored in a desiccator until further evaluation.
Kneaded complex (KC) - The kneaded complex of EPL, $\beta$ $\mathrm{CD}$ and amino acid was prepared by wetting the physical mixture in a mortar with a minimum volume of ethanol/water mixture $(15 / 85, \mathrm{~V} / \mathrm{V})$ and kneading thoroughly with a pestle to obtain a paste, which was then dried under vacuum at room temperature, sieved through a 120 sieve and stored in a desiccator until further evaluation.

\subsection{Fourier transform infrared spectroscopy (FTIR) Analysis}

FTIR spectroscopy was performed on a Fourier Transform Infrared Spectrometer (Alpha T Bruker). Spectra were collected in the $4000-400 \mathrm{~cm}-1$ range.

\subsection{Differential scanning calorimetry (DSC) Analysis}

Thermograms of EPL, $\beta$-CD, ternary complex were recorded on a Mettler Toledo, Staresw 920. Each sample (2-4mg) was heated in crimped aluminum pans at a scanning rate of $10^{\circ} \mathrm{C} / \mathrm{min}$ in an atmosphere of nitrogen using the range of $100-240^{\circ} \mathrm{C}$. The temperature calibrations were performed periodically using indium as a standard.

\subsection{Powder X-ray diffractometry (PXRD) Analysis}

To obtain the changes in the crystallanity of the ternary complex prepared, the PXRD study was carried out by using $X$ ray diffractometer (Miniflex 600 X-Ray Diffractometer Rigaku Corporation Japan). For this the samples of pure drug, $\beta$-CD, and prepared ternary complex were irradiated with monochromatised $\mathrm{CuK} \alpha$ radiation and analyzed between from $5^{\circ}$ to $60^{\circ}(2 \theta)$.

\subsection{Scanning electron microscopy (SEM) Analysis}

The surface morphological features of EPL, binary, ternary complex were investigated using a scanning electron microscope (VEG A3 TESCAN) operated at an acceleration voltage of $20 \mathrm{kV}$ and obtained microphotographs were examined at X500 and X2000 magnifications.

\subsection{Saturation solubility studies}

Saturation solubility studies of EPL, binary and ternary complexes were conducted in triplicate. An excess amount of EPL and complexes were added to $20 \mathrm{~mL}$ of distilled water in vials sealed with stoppers and shaken in rotary flask shaker at room temperature $\left(25 \pm 0.5^{\circ} \mathrm{C}\right)$ for $24 \mathrm{~h}$. A portion of solution was withdrawn, filtered through Whatman filter paper 41 and analyzed spectrophotometrically (Shimadzu UV spectrophotometer 1800) at $390 \mathrm{~nm} .16$

\subsection{Dissolution studies}

In vitro dissolution studies for EPL and its binary and ternary complexes were carried out using USP Type-II dissolution test apparatus (EDT 08LX Electrolab). Sample equal to $100 \mathrm{mg}$ of EPL was used in each test. The dissolution tests were performed using $900 \mathrm{ml}$ of phosphate buffer $\mathrm{pH} 6.8$ at $37 \pm 0.5^{\circ} \mathrm{C}$ with RPM maintained at 100. The release of EPL from each sample was determined by withdrawing $5 \mathrm{ml}$ samples at preset time intervals, and were filtered, appropriately diluted and analyzed spectrophotometrically (Shimadzu UV spectrophotometer 1800) at $390 \mathrm{~nm} .5 \mathrm{ml}$ of fresh medium was transferred to maintain sink conditions.

\subsection{Stability Study}

Stability study binary and ternary complexes of EPL was carried out with the help of (Remi SC-19 Plus) by storing $1 \mathrm{gm}$ of each selected complex in an ambered coloured 
screw capped glass bottles at accelerated and controlled temperatures $40^{\circ} \mathrm{C}$ and relative humidities (75\%) for a period of 3 months. The complexes were evaluated for physical appearance, drug content and in-vitro dissolution at the end of three months. ${ }^{8}$

\section{RESULTS AND DISCUSSION}

\subsection{Phase solubility studies}

The complexing nature of EPL along with $\beta$-CD in water was studied by the phase solubility as per standards set by Higuchi and Connors 1965. Fig.2 shows the phase solubility diagrams of EPL with $\beta$-CD and that of ARG. A typical Bstype solubility profile as stated by Higuchi and Connors classification system was shown by the EPL and $\beta$-CD, binary complex. Bs-type response stands for development of inclusion complexes of limited solubility within the range of $\beta$-CD concentrations examined. The solubility of EPL with $\beta$-CD was higher than pure EPL. The phase solubility profile of EPL in aqueous $\beta$-CD solution in presence of $0.25 \%$ ARG displayed $A_{L}$ type of solubility curve with linear increase in solubility of EPL with increasing the concentration of $\beta-C D$. And slopes curves of phase solubility profile were lesser than 1 characterizing the formation of a 1:1 stoichiometry water soluble complex. 15-18 The values of Ks and CE of complex increased with the incorporation of ARG to the binary complex showing more effectiveness of ternary system over binary. The values of Ks for binary complex and ternary complex were found to be 505.18 and 876.47 respectively. Complexation efficiency of binary complex was found to be 0.24 and for ternary complex it was 0.48 which is almost double of binary complex. Advancement in phase solubility parameters of EPL by complexation with $\beta$-CD in presence of ARG can be attributed to its electrostatic/hydrogen bonding interaction and salt formation with $\beta$-CD and EPL. 19,20

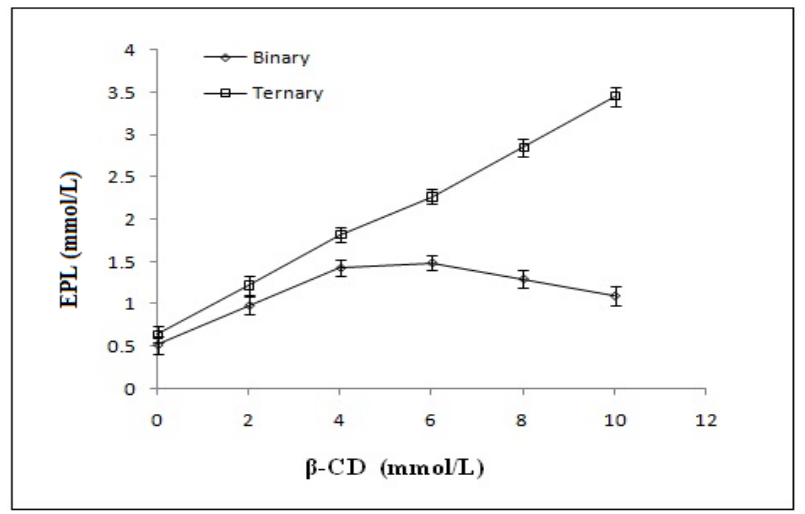

Figure 2: Phase solubility diagrams of EPL: $\beta$-CD (Binary complex) and EPL: $\beta$-CD: ARG (ternary complex) in water.

\subsection{FTIR measurements}

The molecular interaction between EPL and $\beta$-CD was revealed by FTIR. Fig. 3 shows the FTIR spectra of binary and ternary complex. For EPL, principle absorption peaks were found near to 1742, 1673, and $1555 \mathrm{~cm}-1$ from carboxyl, amide, and thiocarbonyl stretching vibrations, respectively. All these principle characteristic peaks were retained and did not shift both in binary and ternary complex. As the results, it is concluded that EPL is dispersed uniformly in the $\beta$-CD.

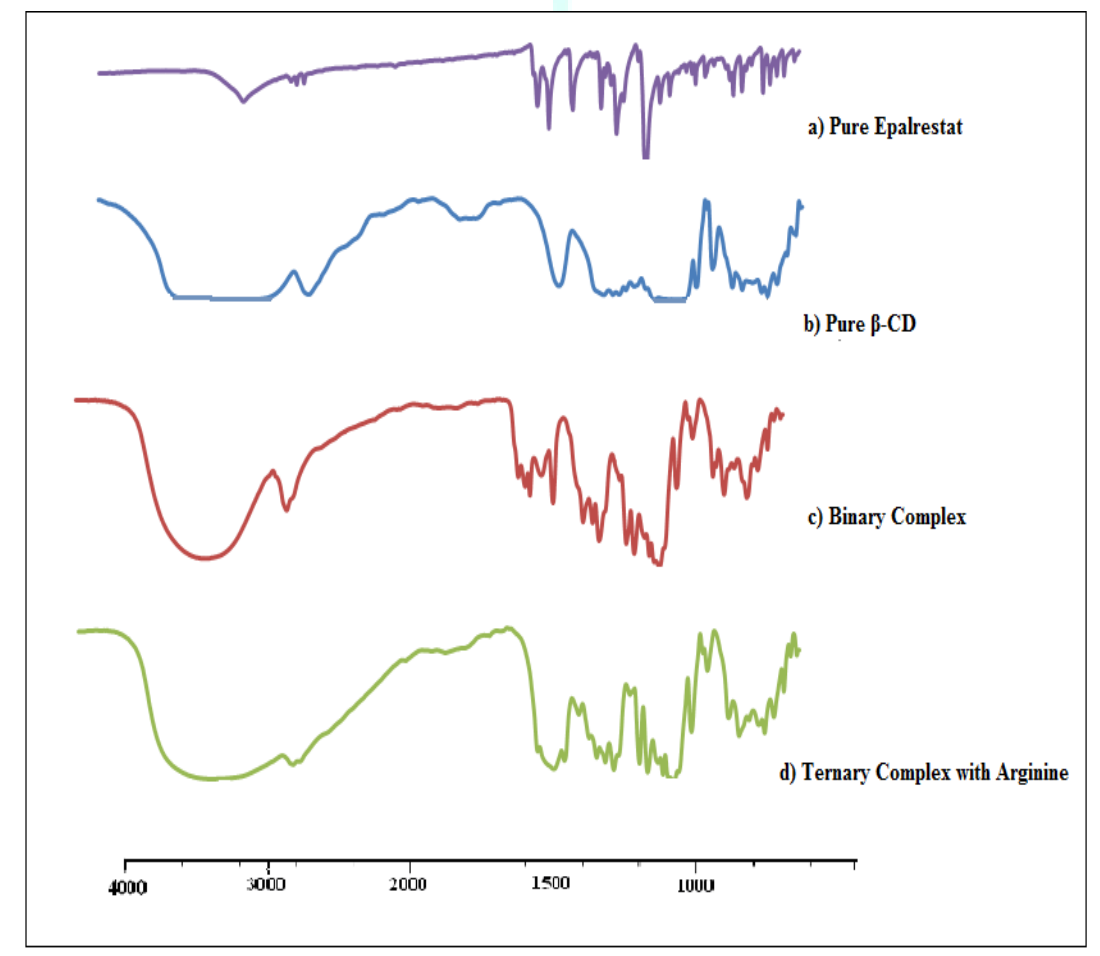

Figure 3: FTIR spectra of A) EPL B) Pure $\beta$-CD C) Binary complex D)EPL: $\beta$-CD:ARG ternary system

\subsection{DSC measurements}

DSC analyses were performed to evaluate the thermal behavior of EPL, $\beta$-CD and ternary complex, respectively (Fig. 4) and interaction between EPL and $\beta$-CD in complex formation. When guest molecule incorporated into cyclodextrin central cavity, their melting point shift to different temperature or it may disappear. One sharp endothermic peak was observed near around $215^{\circ} \mathrm{C}$ contributed to the fusion of crystalline EPL in the ternary thermograms broad endothermic peak was observed near 
around $140^{\circ} \mathrm{C}$, which can be attributed to dehydration during the heating process of the $\beta$-CD. In the ternary complex, the endothermic peak obtained from EPL decreased or almost disappeared. It may be due to low interaction between the components in the ternary complex. These results suggested that EPL and $\beta$-CD were molecularly dispersed in the amorphous form in ternary complex.

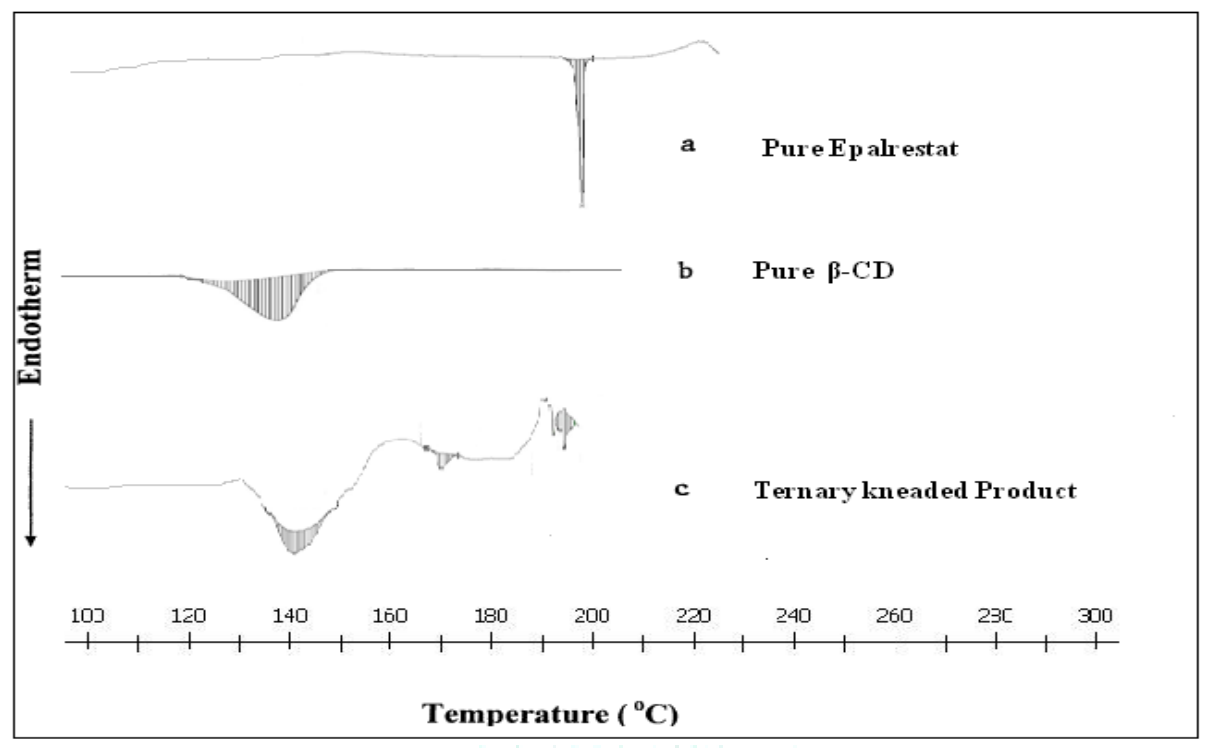

Figure 4: DSC thermograms of A) EPL B) Binary complex C) EPL: $\beta$-CD:ARG ternary system

\subsection{PXRD measurements}

PXRD patterns of EPL, binary and ternary complex (molar ratio 1:1) are represented in Fig. 5. The pattern of the ternary complex could be interpreted as superposition of EPL and $\beta$-CD representing absences of crystalline traces of EPL indicating entrapment of EPL in $\beta$-CD. In ternary systems, the overlapping of $\beta$-CD and ARG crystalline peaks was observed. The PXRD showed a halo pattern and no diffraction peak occurred when EPL was kneaded with $\beta$ $\mathrm{CD}$ at a 1:1 molar ratio. Crystalline EPL turned amorphous by kneading with $\beta$-CD. It was revealed that the crystalline structure of EPL was distorted and EPL molecules were converted into amorphous state.

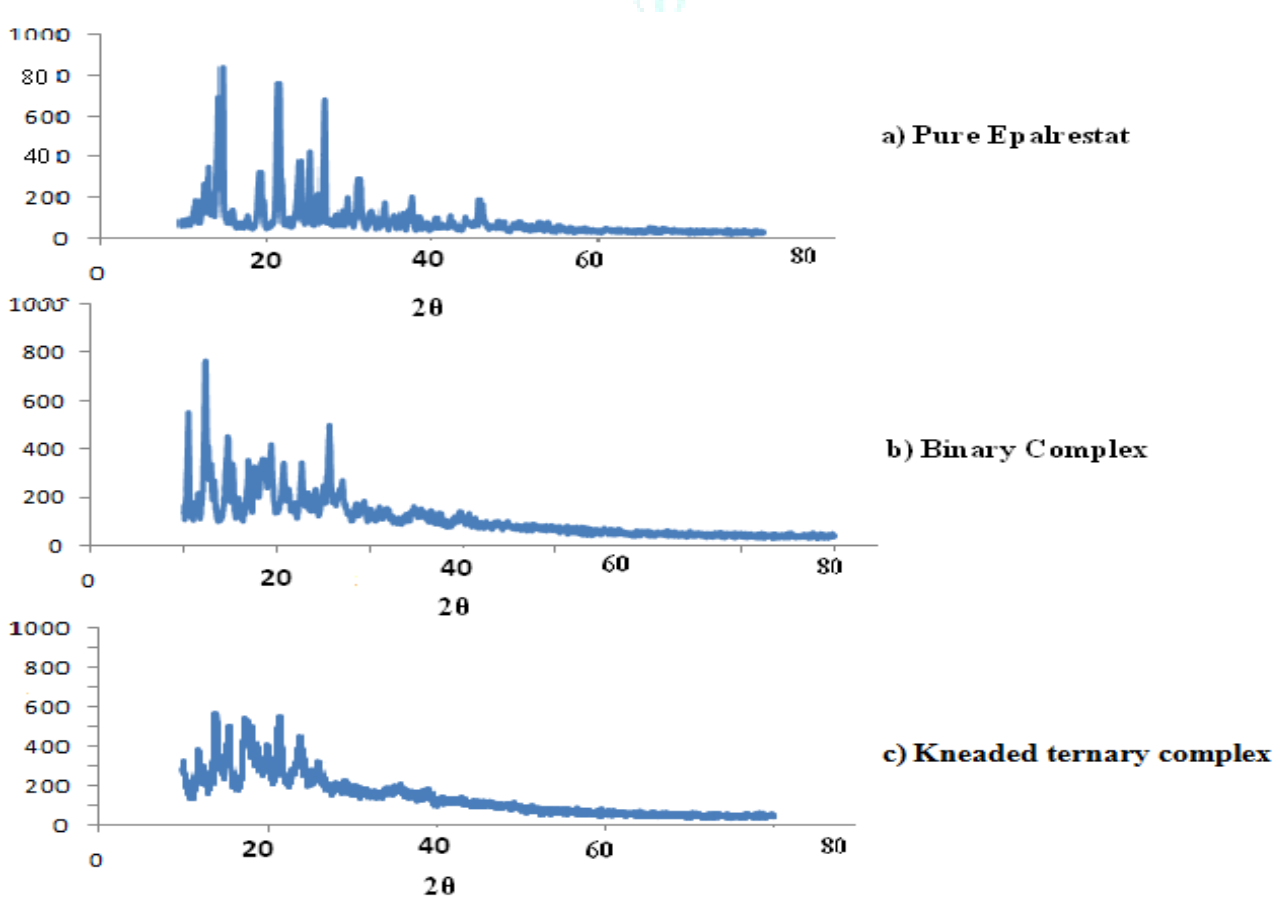

Figure 5: PXRD patterns of A) EPL B) Binary complex C) EPL: $\beta$-CD:ARG ternary complex 


\subsection{SEM analysis}

The surface morphological features of EPL and ternary complexes are shown in Fig. 6. Separate entities of amorphous broken particles of Pure EPL were appeared (Fig. 6A). Binary complex particles exhibited altered shape and agglomerate type morphology (Fig. 6B) due to crystalline nature of $\beta$-CD. Change in morphology of ternary complexes was observed due to the crystalline nature of $\beta$-CD and ARG (Fig. 6C) showing agglomerated crystalline images. The alerted morphology of kneaded complexes particles indicates existence of a single phase within the complex attaining maximum complexation. 21
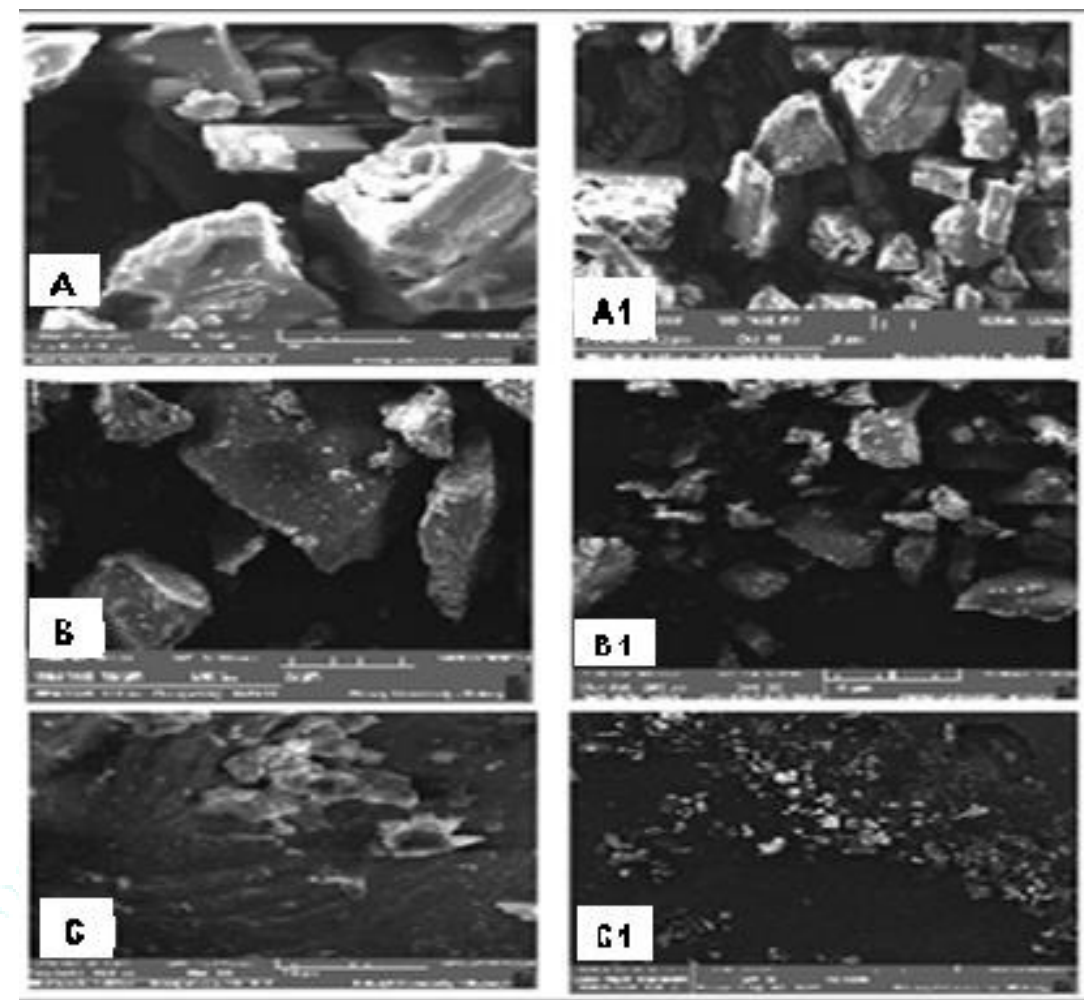

Figure 6: SEM images of A) Pure EPL B) Binary complex C) EPL: $\beta-C D: A R G$ ternary complex

\subsection{Saturation solubility studies}

Remarkable enhancement in the solubility of binary and ternary complex as compared to pure EPL was observed in the saturation solubility studies. Pure EPL exhibited a solubility of $47 \pm 0.04 \mu \mathrm{g} / \mathrm{mL}$ in distilled water. The binary and ternary complexes have shown solubility of $98.7 \pm 0.30$ and $163.09 \pm 0.72 \mu \mathrm{g} / \mathrm{mL}$, respectively. There was $210 \%$ increment in the solubility of binary complex and $347 \%$ increment in the solubility of ternary complex was observed which was almost double than binary complex. Formation of stable complex of EPL and $\beta$-CD, altered surface morphological features of the complexes due to kneading and inclusion into the hydrophobic $\beta$-CD cavity resulted in the enhancement in solubility of complex. ARG imparts significant role as a ternary component resulting in improved performance than binary complex ${ }^{22}$.

\subsection{In vitro dissolution studies}

EPL and its corresponding binary and ternary complexes were evaluated for dissolution properties and compared to the pure EPL. The in-vitro dissolution results were assessed on the basis of cumulative percentage drug release, dissolution efficiency and correlation coefficient (r).

The dissolution curves of EPL and ternary complexes are shown in Fig.7. An enhancement in dissolution profile was observed for solid complexes as compared to pure EPL. The binary complex has shown almost $60 \%$ drug release in $60 \mathrm{~min}$. And ternary complex demonstrated grater dissolution profile as compared to binary complex with $78.18 \%$ drug release within $60 \mathrm{~min}$ in dissolution media. Phase solubility parameters such as Ks and CE were greatly promoted and resulted in the greater fruitfulness of ternary complex. Higher release rate was due to positive effect of addition of basic amino acid ARG.12,20,23 Hence, we can arrive at a judgment that ternary complex of EPL with $\beta-C D$ and ARG could be reliable approach for improved dissolution properties.

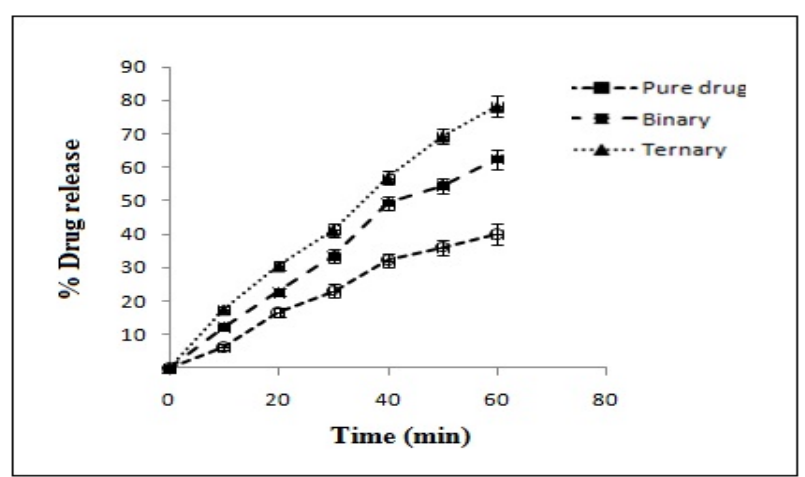

Figure 7: Dissolution profile of pure drug, binary and ternary kneaded complex

\subsection{Stability Study}

There was no significant change in the physical appearance, drug content and percent drug dissolution in 
the EPL complexes. A stability results clearly indicate that the complexes were sufficiently stable under accelerated and controlled conditions.

\section{CONCLUSION}

From the present investigation it is concluded that the formulated complexes revealed the key performance of $\beta$ $\mathrm{CD}$ and ARG to ameliorate the solubility and dissolution rate of EPL and also suggests a significant increase in the

\section{REFERENCES}

1. Takayuki F, Shoma T, Noriko O, Mihoko G, Enhanced dissolution and skin permeation profiles of epalrestat with $\beta$ cyclodextrin derivatives using a cogrinding method, Eur J Pharm Sci, 2017; 106:79-86.

2. Callaghan BC, Cheng HT, Stables CL, Smith AL, Feldman EL, Diabetic neuropathy: clinical manifestations and current treatments, Lancet Neurol, 2012; 11:521-534.

3. Ramirez MA, Borja NL, Epalrestat: an aldose reductase inhibitor for the treatment of diabetic neuropathy, Pharmacotherapy, 2008; 28:646-655.

4. Ohmura C, Watada H, Azuma K, Shimizu T, Kanazawa A, Ikeda F, Yoshihara T, et al, Aldose reductase inhibitor, epalrestat, reduces lipid hydroperoxides in type 2 diabetes, Endocr, J 2009; 56:149-156.

5. Oates PJ, Mylari BL, Aldose reductase inhibitors: therapeutic implications for diabetic complications, Expert Opin Investig Drugs, 1999; 8:2095-2119.

6. Brewster ME, Loftsson T, Cyclodextrins as pharmaceutical solubilizers, Adv Drug Deliv Rev, 2007; 59:645-666.

7. Loftsson T, Duchene D, Cyclodextrins and their pharmaceutical applications, Int J Pharm, 2007; 329:1-11.

8. Mohammed J, Ashok M, Mohammed IK, Ameliorated solubility and dissolution rate of glimepiride by cyclodextrin ternary complexes employing amino acids, Int J Pharm Sci Res, 2017; 8(6):2443-2451.

9. Sapte $\mathrm{S}$, Pore Y, Inclusion complexes of cefuroxime axetil with Bcyclodextrin: Physicochemical characterization, molecular modeling and effect of l-arginine on Complexation, J Pharm Anal, 2016; 6:300-306.

10. Valero M, Perez-Revuelta BI, Rodriguez LJ, Effect of PVP K-25 on the formation of the naproxen: $\beta$-cyclodextrin complex, Int J Pharm, 2003; 253:97-110.

11. Gajare P, Patil C, Kalyane N, Effect of hydrophilic polymer on pioglitazone complexation with hydroxyl propyl bcyclodextrin, Dig J Nanomater Bios, 2009; 4:891-897.

12. El-Maradny HA, Mortada SA, Kamel OA, Hikal AH Characterization of ternary complexes of meloxicam-HP $\beta-C D$ and PVP or L-arginine prepared by the spray-drying technique, Acta Pharm, 2008; 58:455-466. complexation efficiency between EPL and $\beta$-CD by addition of small amounts of amino acid ARG by kneading. Formation of inclusion complexes, greatly enhance the solubility of EPL, which can thus increase its bioavailability and improving pharmaceutical potential of the drug.

\section{ACKNOWLEDGMENT}

Authors are thankful to Zydus Cadila Ahmadabad (India) for providing Epalrestat drug as gift sample.

13. Bramhane DM, Saindane NS, Vavia PR, Inclusion complexation of weakly acidic NSAID with b-cyclodextrin: selection of arginine, an amino acid, as a novel ternary component, J. Incl. Phenom, Macrocycl. Chem 2011; 69:453-460.

14. Maheshwari DG, Chaudhari Kl, Development and Validation of UV Spectrophotometric Method for Simultaneous estimation of Epalrestat and Methylcobalamin in the Pharmaceutical Dosage Form, Int J Pharmtech Res, 2014; 6(4):1180-1188.

15. Higuchi T, Connors KA, Phase-solubility techniques, Adv Anal Chem Instr, 1965; 4:117-212.

16. Jadhav P, Pore Y, Physiochemical Thermodynamic and analytical studies on binary and ternary inclusion complex of bosentan with hydroxyl - B-cyclodextrin, Bulletin of Faculty of Pharmacy Cairo University, 2017; 55:147-154.

17. Sherje AP, Kulkarni V, Murahari M, Nayak UY, Bhat P, Suvarna $\mathrm{V}$, et al, Inclusion Complexation of Etodolac with Hydroxypropyl-betacyclodextrin and Auxiliary Agents: Formulation Characterization and Molecular Modeling Studies, Mol Pharm, 2017; 3:14(4):1231-1242.

18. Ribeiro L, Loftsson T, Ferreira D, Investigation and physicochemical characterization of vinpocetine-sulfobutyl ether b -cyclodextrin binary and ternary complexes. Chem Pharm Bull 2003; 51:914-922.

19. Patil A, Pore Y, Kuchekar B, Effect of L-arginine on bicalutamide complexation with hydroxypropyl-bcyclodextrin, Dig J Nanomater Bios, 2008; 3:89-98.

20. Jadhav P, Pethkar B, Pore Y, Physicochemical and molecular modeling studies of cefixime-L-arginine-cyclodextrin ternary inclusion compounds, Carbohydr Polym, 2013; 98:1317-1325.

21. Fernandes CM, Vieira MT, Veiga FJ, Physicochemical characterization and in vitro dissolution behavior of nicardipine-cyclodextrins inclusion compounds, Eur J Pharm, 2002; 15(1):79-88.

22. Shinde VR, Shelake MR, Shetty SS, Enhanced solubility and dissolution rate of lamotrigine by inclusion complexation and solid dispersion technique, J Pharm Pharmacol, 2008; 60:1121-1129.

23. Laveneziana D, Speranzam R, Raullim P, Ibuprofen - arginine in the management of pain, Clin Drug Invest, 1996; 11:1-7. 